\title{
Leading from the Middle: Practical Innovation in Public Mental Health
}

\author{
Allison N. Ponce ${ }^{1} \cdot$ Kyle W. Pedersen ${ }^{1} \cdot$ Michael J. Sernyak $^{1}$ \\ Received: 1 October 2021 / Accepted: 8 January 2022 / Published online: 28 January 2022 \\ (c) The Author(s), under exclusive licence to Springer Science+Business Media, LLC, part of Springer Nature 2022
}

\begin{abstract}
Leaders in public mental health are responsible for ensuring the care environment is conducive to provider wellbeing, and ultimately patient care. Given the effects of stress and burnout, healthcare organizations must explore interventions to support their employees. The Leadership + Innovation Lab is a pilot project focused on enhancing leadership skills, innovation capacity, and peer connections among clinical managers. Participants executed individual or group projects to improve the care environment and co-created a peer consultation program. They reported increased connection with peers, innovation and leadership skills, and capacity to facilitate a better experience for their provider staff as a result of the program. This model can be used in other settings to help achieve the goals of the Quadruple Aim and improve communication.
\end{abstract}

Keywords Public mental health · Provider wellness · Quadruple Aim

In public mental health centers, the relentless flow of work can negatively affect providers, stifle creativity, and impede teamwork. Resource scarcity can lead to burnout (Rondeau et al., 2005) and turnover (Campbell et al., 2013) which may interrupt attempts to provide the best clinical care and optimal functioning of the system. While the Triple Aim of healthcare focuses on improving the experience of care and health of populations while reducing costs (Berwick et al., 2008), the more recently defined Quadruple Aim has emerged, focusing on enhancing the experience of the care provider (Bodenheimer \& Sinsky, 2014; Sikka et al., 2015).

When healthcare professionals experience support for inclusive leadership, coordination, collegial support, and enhancing resilience, they may experience higher job satisfaction, lower levels of burnout, and increased innovation (Havens et al., 2018; Moffatt-Bruce et al., 2019; Wang et al., 2019). Efforts to address problems such as stress and burnout show that a combination of individual and organizational interventions may provide the most durable positive results (Awa et al., 2010). The mental health field has seen efforts to improve the quality of experiences for various stakeholders including clients (Cook et al., 2019) and informal caregivers

Allison N. Ponce

allison.ponce@yale.edu

1 Department of Psychiatry, Connecticut Mental Health Center, Yale School of Medicine, New Haven, CT 06519, USA of individuals with serious mental illness (Udechuku et al., 2015). It is important that mental health professionals are included in these efforts.

Leaders in public mental health systems are well-positioned support the wellbeing of mental health providers, which the literature would suggest could lead to improvements in care. Offering organizational mechanisms for increasing providers' sense of control and collegial support enhances the experience of caregiving. Given that empowerment in organizations is positively correlated with innovation capability (Cakar \& Ertürk, 2010), efforts to empower staff with decision making opportunities and enhance collaboration could lead to an increase in innovative ideas and a more satisfied workforce, and ideally, a better experience for patients.

The Connecticut Mental Health Center (CMHC) has focused on workforce engagement as a means of achieving this fourth aim related to provider experience, specifically with regard to innovation, teamwork, communication, and leadership development. It is increasingly important for community mental health centers to promote leadership and a culture of innovation that continuously generates and tests transformative ways to improve quality of the work experience and care. What if we intervened on the system by injecting low-cost energy and resources in the middle layer of the structure? Could this stimulate better and more open thinking to create a new mechanism for change and 
improvement in the care experience? Could it offer opportunities for better communication?

CMHC is an academic-public partnership between the State of Connecticut Department of Mental Health and Addiction Services and the Yale Department of Psychiatry in New Haven, CT. The CMHC serves about 4000 individuals with low income annually and is staffed by hundreds of state and university employees supporting recovery and providing mental health and substance use treatment in both inpatient and outpatient settings.

Among this large staff group, CMHC clinical managers were identified as key stakeholders well-positioned to influence structural concerns and services as well as understand and represent the perspectives of their direct reports. These middle managers occupy a critical space in the organization as people who can facilitate communication between different parts of the system, are close to the clinical care but also understand administrative concerns, and are invested in the wellbeing of their staff. With this pivotal group in mind, the interprofessional Leadership + Innovation $(\mathrm{L}+\mathrm{I})$ Lab was created. This project focused on strengthening peer support and workplace satisfaction among participants while increasing leadership and idea-generating capacity among a team of potentially influential personnel.

Nine of the 11 eligible managers joined the project; they were social workers, occupational therapists, nurses, and rehabilitation professionals who oversaw teams or departments that range in size from a few employees to dozens serving both inpatient and outpatient client populations. The guiding principle —in the spirit of a learning lab-was to use data from the workplace to generate meaningful activities that were experiential, project-based, iterative, and immediately applicable. These projects were to be viewed as interventions on the system-changes that may ultimately enhance the experience of providing and receiving care.

With charitable funds from the CMHC Foundation and internal personnel resources, we designed an initial sevenmonth program with core elements related to innovation and idea development, project management, peer consultation, and team-building. Explicit efforts to create a productive and enjoyable experience for participants included meeting offsite and providing meals during meetings. These acts were intended to give care to the caregivers and limit interruptions from their daily work environment.

The day-long opening session and subsequent half-day monthly sessions focused on identification and exploration of leadership styles and building community among the managers. Throughout the intervention, individual, small group, and large group exercises encouraged and stimulated participants' capacity to generate ideas, create innovative solutions to problems, and effectively manage projects. Highly participatory sessions were led by a project management consultant and a psychologist who helped the group initiate their peer consultation process. The penultimate session was attended by the CMHC CEO who presented an organizational case study related to managing patient flow in the system. He engaged with the participants in several idea-generating and priority setting activities they learned over the course of the program. This activity provided the opportunity for participants to practice new skills and thinking regarding an organization problem affecting them all. While this discussion was considered a proof-of-concept activity, this mechanism could be developed as an active problem-solving tool for the organization.

A cornerstone of the $\mathrm{L}+\mathrm{I} \mathrm{Lab}$ was individual or paired projects to improve the experience of providing care. The cycle of mental health services typically involves patients entering the system, engaging in care, then, hopefully, being discharged. However, at MHC large caseloads are the norm and with thinly-stretched resources, staff attrition, and provider stress, the $\mathrm{L}+\mathrm{I}$ Lab participants were challenged to identify "stuck points" in their environments that interfere with staff wellbeing and patient experience. These were used as the starting points for testing new ideas and utilizing project management principles to improve the system.

The projects highlighted the many ways that relatively simple interventions can have enormous impact on staff and patients. One participant managed a program with her staff to distribute donated bicycles to patients to enhance their health and transportation access. Another spearheaded an effort to improve the physical environment of their clinic. A new team leader facilitated a team-building retreat, and two other participants collaboratively addressed caseload sizes by enhancing group treatment programming. A seasoned supervisor created a discipline-specific peer consultation group consisting of colleagues in her area across departments while another designed a new treatment planning process for her unit. Yet another created a mechanism to advertise a case-management service available to clients without a referral. These initiatives would not have carried the same meaning or impact had they been dictated by the organization's leadership, rather, they emerged from the participants' knowledge of their teams and patients. They were empowered to think creatively and act decisively. $\mathrm{L}+\mathrm{I}$ Lab consultation resources were utilized to help the managers workshop ideas, define discrete goals and steps, and set realistic timelines and measures of success. Resources were made available to fund the projects, though the majority of projects did not require any financial support. In addition to burnishing the participants' skills, each project had the benefit of helping staff and clients, further increasing the benefit to the managers.

At the end of the intervention, the vast majority of participants reported feeling like more effective leaders and more able to improve their staffs' experience of providing care as a result of the program. They self-reported an increase 
in workplace satisfaction and unanimously reported the program enhanced peer support among participants, and resulted in increased innovation, leadership capacity, and capacity to develop ideas. These results should be interpreted with caution given the small number of participants but they do appear to indicate that the participants had a positive experience. Sustained benefits 18 -months postprogram included an ongoing monthly peer support group and reports of improved capacity for having difficult discussions such as negotiations about new patient placements and supervising staff.

Though not an initial goal of the project, the structure of the L+I Lab has offered an unprecedented, ongoing mechanism for communication during the COVID-19 pandemic. The existing group structure and the connection to the leaders supported the gathering of information to support efforts to protect and enhance wellbeing among staff at CMHC. The first and second authors of this article are the Chief Wellness Officers for CMHC and have utilized the relationship and group structure from the $\mathrm{L}+\mathrm{I} \mathrm{Lab}$ to collect information about the staff experience to design interventions during this time of incredible stress and anxiety. Early on, this included soliciting informal feedback from the participants about their teams' needs during the crisis, and expanded to visits to their virtual team meetings for direct discussion with staff. In addition to maintaining strong lines of communication with the participant group, the future of the L+ I Lab includes ongoing, periodic reunions to continue working on skills and planning additional group-based projects.

As mental health leaders consider improving the experience of providing care in the public mental health settings in line with the Quadruple Aim, several lessons can be gleaned from CMHC's inaugural L+I Lab. The first is that offering a thoughtful, supportive educational experience to clinical managers appears to lead to several positive selfreported outcomes. Given the deleterious effects of burnout in healthcare settings and the relatively little formal training and support for mid-level managers, the positivity from the participants speaks to the possibility of addressing an unmet need. Additional benefits include having a positive, invigorated group of middle-managers to connect with in times of crisis (e.g., COVID-19 pandemic) or to convene to discuss ways to improve the experience of work and patient care.

This project benefited from strong personnel resources to design and implement the intervention and the availability of financial resources to fund projects as needed. However, even with relatively modest financial resources, simple programs can support and educate managers to identify concrete improvements to the provider experience and to encourage peer support. Further consideration is being given to how participants can use their newly-developed skills to teach their teams new concepts and approaches to their work. The project represents an opportunity for continuing education and is housed in the CMHC Education Department, however other homes could be appropriate such as offices of medical and professional staff or human resources. The possibility of clinical quality improvement projects will be an important future consideration, perhaps with the participants serving as a formal quality improvement group. While this first iteration of the $\mathrm{L}+\mathrm{I}$ Lab focused on clinical managers, there are many other staff constellations that could benefit from such education and support. These include non-clinical managers who could improve their work products and wellbeing with increased capacity to generate ideas and manage projects.

The risks to the mental health workforce brought on by provider stress and burnout are significant. Public mental health leaders must take responsibility for supporting their staff and improving their experience of providing care to reduce distress, increase retention, improve the effectiveness of managers, and ultimately, increase the quality of treatment. The L+I Lab represents one mechanism for addressing these crucial workforce issues with an eye toward improving the care experience for patients as well.

Funding This work was funded in part by the State of Connecticut, Department of Mental Health and Addiction Services, but this publication does not express the views of the Department of Mental Health and Addiction Services or the State of Connecticut. The views and opinions expressed are those of the authors. The project detailed here was funded in part by a charitable contribution to the Connecticut Mental Health Center Foundation from the Otsuka Sakura Independent Medical Education and Charitable Contributions module.

\section{References}

Awa, W. L., Plaumann, M., \& Walter, U. (2010). Burnout prevention: A review of intervention programs. Patient Education and Counseling, 78, 184-190. https://doi.org/10.1016/j.pec.2009.04.008

Berwick, D. M., Nolan, T. W., \& Whittington, J. (2008). The Triple Aim: Care, health and cost. Health Affairs, 27, 759-769. https:// doi.org/10.1377/hlthaff.27.3.759

Bodenheimer, T., \& Sinsky, C. (2014). From Triple to Quadruple aim: Care of the patient requires care of the provider. The Annals of Family Medicine, 12(6), 573-576. https://doi.org/10.1370/afm. 1713

Çakar, N. D., \& Ertürk, A. (2010). Comparing innovation capability of small and medium-sized enterprises: Examining the effects of organizational culture and empowerment. Journal of Small Business Management, 48(3), 325-359.

Campbell, N. S., Perry, S. J., Maertz, C. P., Allen, D. G., \& Griffeth, R. W. (2013). All you need is resources: The effects of justice and support on burnout and turnover. Human Relations, 66(6), 759-782. https://doi.org/10.1177/0018726712462614

Cook, J. A., Shore, S., Burke-Miller, J. K., Jonikas, J. A., Hamilton, M., Ruckdeschel, B., \& Bhaumik, D. (2019). Mental health selfdirected care financing: Efficacy in improving outcomes and controlling costs for adults with serious mental illness. Psychiatric Services, 70(3), 191-201. https://doi.org/10.1176/appi.ps.20180 0337 
Havens, D. S., Gittell, J. H., \& Vasey, J. (2018). The impact of relational coordination on nurse job satisfaction, work engagement and burnout: Achieving the Quadruple Aim. The Journal of Nursing Administration, 48(3), 132-140. https://doi.org/10.1097/NNA. 0000000000000587

Moffatt-Bruce, S. D., Nguyen, M. C., Steinberg, B., Holliday, S., \& Klatt, M. (2019). Interventions to reduce burnout and improve resilience: Impact on a health system's outcomes. Clinical Obstetrics \& Gynecology, 62(3), 432-443. https://doi.org/10.1097/grf. 0000000000000458

Rondeau, K. V., \& Francescutti, L. H. (2005). Emergency department overcrowding: The impact of resource scarcity on physician job satisfaction. Journal of Healthcare Management, 50(5), 327-341. https://doi.org/10.1097/00115514-200509000-00009

Sikka, R., Morath, J. M., \& Leape, L. (2015). The Quadruple Aim: Care, health, cost and meaning in work. BMJ Quality \& Safety, 24, 608-610. https://doi.org/10.1136/bmjqs-2015-004160
Udechuku, A., Mayo-Wilson, E., Harrison, B., Shiers, D., Woodhams, P., Kuipers, E., \& Kendall, T. (2015). Interventions to improve the experiences of caring for people with severe mental illness: Systematic review and meta-analysis. British Journal of Psychiatry, 206(4), 268-274. https://doi.org/10.1192/bjp.bp.114.147561

Wang, Y. X., Yang, Y. J., Wang, Y., Su, D., Li, S., Zhang, T., \& Li, H. (2019). The mediating role of inclusive leadership: Work engagement and innovative behaviour among Chinese head nurses. Journal of Nursing Management, 27(4), 688-696. https://doi.org/10. 1111/jonm.12754

Publisher's Note Springer Nature remains neutral with regard to jurisdictional claims in published maps and institutional affiliations. 